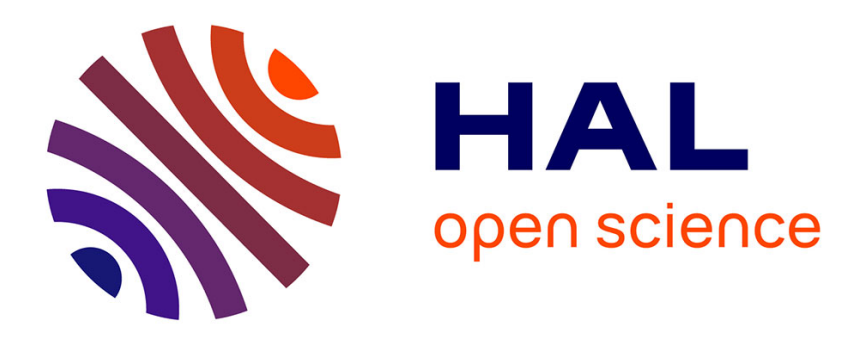

\title{
Pourquoi faut-il parler de la mort des animaux de refuge?
}

Jérôme Michalon

\section{To cite this version:}

Jérôme Michalon. Pourquoi faut-il parler de la mort des animaux de refuge?. Etudes sur la mort. Revue de la Société de thanatologie, 2014, 145 (145), pp.73-82. 10.3917/eslm.145.0073 . halshs01095439

\section{HAL Id: halshs-01095439 \\ https://shs.hal.science/halshs-01095439}

Submitted on 12 Nov 2020

HAL is a multi-disciplinary open access archive for the deposit and dissemination of scientific research documents, whether they are published or not. The documents may come from teaching and research institutions in France or abroad, or from public or private research centers.
L'archive ouverte pluridisciplinaire HAL, est destinée au dépôt et à la diffusion de documents scientifiques de niveau recherche, publiés ou non, émanant des établissements d'enseignement et de recherche français ou étrangers, des laboratoires publics ou privés. 


\title{
POURQUOI FAUT-IL PARLER DE LA MORT DES ANIMAUX DE REFUGE ?
}

\author{
Jérôme Michalon
}

L'Esprit du temps | Études sur la mort

\author{
2014/1 - $\mathrm{n}^{\circ} 145$ \\ pages 73 à 82
}

ISSN 1286-5702

Article disponible en ligne à l'adresse:

http://www.cairn.info/revue-etudes-sur-la-mort-2014-1-page-73.htm

Pour citer cet article :

Michalon Jérôme, «Pourquoi faut-il parler de la mort des animaux de refuge ? »,

Études sur la mort, 2014/1 n 145, p. 73-82. DOI : 10.3917/eslm.145.0073

Distribution électronique Cairn.info pour L'Esprit du temps.

(c) L'Esprit du temps. Tous droits réservés pour tous pays.

La reproduction ou représentation de cet article, notamment par photocopie, n'est autorisée que dans les limites des conditions générales d'utilisation du site ou, le cas échéant, des conditions générales de la licence souscrite par votre établissement. Toute autre reproduction ou représentation, en tout ou partie, sous quelque forme et de quelque manière que ce soit, est interdite sauf accord préalable et écrit de l'éditeur, en dehors des cas prévus par la législation en vigueur en France. II est précisé que son stockage dans une base de données est également interdit. 


\title{
POURQUOI FAUT-IL PARLER DE LA MORT DES ANIMAUX DE REFUGE ?
}

\author{
Jérôme MICHALON
}

En France, comme dans de nombreux pays occidentaux, les fourrières municipales sont chargées de recueillir les animaux domestiques (chiens et chats) «en divagation» sur l'espace public, de tenter de retrouver leur propriétaire s'ils en ont un, et d'euthanasier les animaux sans maîtres et ceux considérés comme dangereux. Les associations de protection animale, par le biais de leurs refuges, travaillent en partenariat étroit avec les fourrières pour optimiser la recherche des propriétaires et, le cas échéant, en trouver de nouveaux pour certains animaux. En plus de leurs propres entrées d'animaux (par le biais des abandons d'animaux, ou d'animaux maltraités saisis), les refuges absorbent également une partie des animaux récupérés par les fourrières ; parfois le service de fourrière est même délégué aux associations. Dans les refuges comme dans les fourrières, le recours à l'euthanasie des animaux, par injection létale, est avéré (préconisé dans le cas des fourrières). Mais que sait-on de ces euthanasies ? Pas grand-chose. J'aimerais évoquer ici en quoi il serait important de développer une meilleure connaissance de cette pratique pour mieux comprendre les évolutions récentes de notre rapport aux animaux domestiques.

\section{GESTIONETPROTECTION DES ANIMAUX DOMESTIQUES: UNCOU- PLE INDISSOCIABLE}

Vues d'aujourd'hui, les relations que les sociétés occidentales entretiennent avec les animaux domestiques semblent polarisées autour de deux grandes figures: l'animal dit de compagnie et l'animal de rente. D'un côté, chiens et 
chats, choyés, anthropomorphisés, personnifiés, intégrés dans les familles, mis à l'abri de la souffrance et de la mort. De l'autre, veaux, vaches, cochons, parqués dans des exploitations de moins en moins familiales, destinés à des abattoirs de plus en plus industrialisés, dans lesquels les animaux n'existent que pour leur chair. Cet état de fait témoignerait ainsi de deux dynamiques sociohistoriques distinctes : 1'une visant à rationaliser au maximum la gestion de la vie (et de la mort) animale, dans une optique utilitaire; l'autre s'inscrivant dans une démarche bienveillante vis-à-vis de l'animal en tant qu'individu, dont la valeur est avant tout affective. Pourtant, les travaux sur l'histoire de la protection animale en Europe occidentale et en Amérique du Nord montrent bien que ces deux dynamiques sont intimement liées : le fait que la première loi de protection animale française ait été votée avant tout pour protéger les chevaux des mauvais traitements de leurs charretiers renseigne sur l'origine commune de la volonté de gestion et de la volonté de protection des animaux. La question de l'errance canine au $\mathrm{XIX}^{\mathrm{e}}$ siècle témoigne bien de cette articulation (Baratay, 2011). Le développement des fourrières municipales et d'un système de ramassage et de mise à mort des chiens errants répondait à une exigence de gestion d'une population canine «sauvage» devenue trop gênante dans les milieux urbains. La création des refuges de protection animale, à partir de 1870 en France, constituera une alternative à la mise à mort systématique de ces animaux sans maîtres (ou dont les maitres ne pouvaient pas être identifiés), en les proposant à l'adoption. Grâce au refuge, les associations de protection animale transforment des animaux errants et/ou maltraités en animaux de compagnie le plus souvent, «placés» durablement dans des familles aimantes. Ce faisant, ces associations deviennent des pourvoyeurs importants d'animaux de compagnie, concurrents directs des éleveurs. Les chiffres manquent pour évaluer précisément l'ampleur de la contribution des refuges à l'augmentation de la démographie des animaux de compagnie, mais on peut estimer qu'elle représente une part très importante. Pourtant, même si la vocation première du refuge est bien de trouver un nouveau maître à l'animal, tous les animaux qui y transitent ne connaissent pas cette félicité : dès leur création, les refuges pratiquent la mise à mort des animaux n'ayant pas ou peu d'espoir de sortir du refuge, soit pour des raisons médicales, d'âge, ou tout simplement d'offre et de demande (selon les époques, certaines races de chien sont plus demandées que d'autres). L'euthanasie est ici liée à la nécessité de gérer la population animale à l'intérieur du refuge, pour que le séjour des animaux ne soit pas assimilé à de la maltraitance. Des chiens ou des chats, entassés dans des cages exigus, ayant peu de contacts avec des animaliers débordés de travail: le tableau ne serait pas très flatteur pour des associations affirmant avoir à cœur les intérêts des animaux. L'euthanasie vient apporter une réponse pragmatique à la surpopulation qui guette tout refuge et qui pèse ainsi sur l'image donnée 
de l'action protectrice. On le voit donc, au plan historique, souci de protection et nécessité de gestion se sont construits mutuellement et sont aujourd'hui encore fortement intriqués dans le fonctionnement quotidien des refuges.

\section{UN «TROU NOIR STATISTIQUE ». OÙ SONT LES CHIFFRES ?}

L'association entre gestion et protection apparaît souvent comme un oxymore; dont les euthanasies pratiquées dans les refuges sont les plus beaux exemples. Dans un article sur la SPA de Gennevilliers, le journaliste Philippe Cohen ne manque d'ailleurs pas de souligner ce «paradoxe effrayant» lorsqu'il explique qu' «hormis les abattoirs, l'institution qui tue le plus d'animaux en France est une association de défense des bêtes.» (Cohen, 1984 ; p. 181). Cette affirmation ne s'accompagne malheureusement pas des références ad hoc permettant de vérifier sa véracité. Nous touchons là un point sensible: où sont les chiffres concernant les euthanasies dans les refuges, et au-delà, dans les fourrières? Après avoir effectué plusieurs recherches, il apparaît clairement que, concernant la France, le nombre de ces mises à mort décidées est extrêmement difficile à trouver. Pourtant, la responsabilité des fourrières animales relève des collectivités territoriales, et même si leur gestion est parfois déléguée à des entreprises privées, il serait logique que les autorités disposent des chiffres des euthanasies, et les rendent publics. Comme toute activité de service public, la gestion de l'errance animale doit bien faire l'objet d'un suivi et d'une évaluation, ne serait-ce qu'économique. De leur côté, les associations de protection animale ne communiquent pas ou très peu sur cette question, si ce n'est pour annoncer qu'elles cherchent à réduire la pratique voire même à l'abandonner ${ }^{1}$. De la même façon que le nombre d'adoptions réalisées dans les refuges, le nombre d'euthanasie est un point aveugle pour quiconque souhaiterait constituer une approche démographique de la population des chiens et des chats (puisqu'il s'agit d'eux essentiellement) en France. Dans d'autres pays, la situation est différente, même si l'accès aux chiffres n'a pas été toujours été aisé.

Pour le cas des Etats-Unis, Peter Marsh (2010) explique que l'opinion publique a commencé à s'alarmer de la recrudescence des euthanasies dans les refuges de protection animale dès les années 1970. Malgré cette inquiétude grandissante, il a fallu attendre plusieurs dizaines d'années pour que l'on ait une vision un peu plus précise du phénomène, de ses causes et des moyens pour y remédier. En 1992, Andrew Rowan parle même d'un «trou

1.La Fondation 30 Millions d'Amis soutient financièrement des refuges qui cherchent à limiter le recours à l'euthanasie. Voir : http ://www.30millionsdamis.fr/acces-special/presse/analyse-desabandons.html 
noir statistique» pour évoquer la question de la surpopulation dans les refuges. Dès lors, sous l'impulsion des vétérinaires et de l'industrie des animaux de compagnie, des études sont lancées visant à mieux connaître la démographie des chiens et des chats domestiques sur le territoire américain, en s'intéressant plus spécifiquement aux refuges. Ces «shelter survey» ont permis de connaître tout à la fois le nombre d'animaux entrant dans les refuges, le nombre d'adoption et le nombre d'euthanasies. Le premier enseignement de ces études est que l'unité de mesure est le million: plusieurs millions de chiens et de chats sont mis à mort chaque année dans les refuges américains. Le second enseignement est la baisse globale du recours à l'euthanasie: d'une estimation de 23,4 millions d'euthanasie de chiens et de chats en 1970, on passe à 4,9 millions pour l'année 1997 ; chiffres qui depuis se stabilisent autour de 4,2 millions par an. Le troisième enseignement tient à la connaissance des facteurs favorisant le recours à l'euthanasie. En l'occurrence, il y a une corrélation forte entre le taux d'admission dans le refuge et son taux d'euthanasie; dit autrement, plus le refuge sélectionne les animaux qu'il accueille (en refusant les abandons de «convenance» par exemple), moins il aura recours à l'euthanasie. La maîtrise des flux démographiques internes au refuge serait donc la clé pour réduire le nombre d'euthanasies. De fait, ces études mettent en lumière l'intérêt de développer le stock de connaissances sur ce qui se passe dans les refuges. Sortis du trou noir, des prises s'offrent aux acteurs pour mettre en œuvre une gestion «éclairée » de la population animale.

\section{MONTRER LA MORT POUR REDONNER DU SENS AU TRAVAIL EFFECTUÉ DANS LES REFUGES}

Néanmoins, mon propos n'est pas particulièrement d'appeler de mes vœux cette gestion «evidence-based », ni même de militer pour une réduction du recours à l'euthanasie dans les refuges. Il s'agit plutôt d'insister sur l'importance de simplement rendre visible la question. Lever le voile sur l'opacité qui règne actuellement en France sur ces pratiques aurait en effet comme vertu de redonner du sens au travail des salariés et des bénévoles qui œuvrent quotidiennement dans les refuges. Ayant observé ce travail de l'intérieur (Michalon, 2013), j'ai pu me rendre compte de sa complexité et des dilemmes qu'il génère chez les employés du refuge. J'ai constaté leur agacement face à des maîtres venant faire piquer leur vieux chien parce qu'au refuge c'est moins cher que chez le vétérinaire. J'ai entendu leur lassitude face à une activité routinière qu'ils pensent parfois déconnectée de la cause animale. J'ai écouté leur irritation de voir le refuge confondu avec une déchetterie pour animaux. J'ai ressenti leur abattement lorsqu'ils faisaient état de ce 
flux ininterrompu d'animaux entrant au refuge. Bref, j'ai pu voir comment la gestion pesait sur la protection. Pour autant, il m'a également été donné de voir la manière dont les deux s'interpénétraient et s'articulaient. D'une part, la pratique de l'euthanasie - paroxysme de la tension entre gestion et protection - génère des conflits entre les salariés et les bénévoles des refuges, entre les salariés eux-mêmes (A-t-on raison de proposer tel animal plutôt qu'un autre à l'euthanasie ?). La récurrence de ces conflits témoigne d'un rapport à l'activité qui n'est pas si routinisé, banalisé, qu'on veut bien le dire : malgré les critères qu'ils se donnent pour choisir quels animaux seront euthanasiés (âge, « race », comportement, «plaçabilité » potentielle), les employés du refuge ne les appliquent pas automatiquement, sans réfléchir. Ils se posent des questions, discutent et justifient leurs décisions au cas par cas. Et quand une querelle éclate entre un bénévole qui reproche à un salarié d'avoir «fait partir» tel chien un peu trop tôt, c'est au nom de la cause protectrice que chacun défend son point de vue. De fait, d'un point de vue pragmatique, le recours à l'euthanasie permet de rester en contact avec le cœur de la cause, de rappeler ce qui est important pour le groupe d'individus réunis dans le refuge ; et ainsi, de se situer au plus près de la responsabilité qu'implique la prise en charge du destin et de la vie de certains individus animaux. Ne pas montrer l'aspect gestionnaire du refuge ne permet pas de rendre compte de ce que représente la tâche de celles et ceux qui consentent à accepter cette charge: montrer l'euthanasie - ne serait ce que comme potentialité - redonne de la consistance à l'activité protectrice. D'autre part, rendre visibles les euthanasies, leur nombre et leur condition, semble être un bon moyen de mettre en lumière l'enjeu concret du refuge: la diffusion à grande échelle - par le biais de l'adoption - d'êtres singuliers et irremplaçables. Car il faut bien comprendre que le séjour dans le refuge marque pour les animaux une étape importante: ils acquièrent une nouvelle identité, souvent un nouveau nom, un nouveau propriétaire (ils appartiennent légalement à l'association - même après l'adoption), une nouvelle histoire. Sortant du refuge, les voilà devenus des « vivant-personne (Micoud, 2010), avec une existence sociale propre, dont on reconnaît le caractère unique et irremplaçable. Tout le travail du refuge est donc d'insérer certains animaux qui y transitent dans la communauté humaine : c'est donc un travail anthropologique qui s'accomplit ici, à travers l'accueil de nouveaux êtres dans l'humanité comme communauté, et non comme espèce. A-t-on besoin de montrer la mort pour éclairer cette dimension anthropologique du refuge? Peut-être. 


\section{DELA VALEUR ANTHROPOLOGIQUEDU REFUGECOMMEMATRICE}

En effet, j'ai pu observer que le refuge, par son organisation spatiale et par la division du travail qui y régnait, permettait d'opérer des distinctions ontologiques importantes à propos d'un même animal. En caricaturant, tel chien vu par les secrétaires sera avant tout une individualité symbolique (une biographie singulière), alors que l'animalier s'attachera plutôt à analyser son comportement ici et maintenant sur un mode éthologique ; enfin, la personne qui prendra en charge le chien dans l'infirmerie le verra plutôt comme une matière biologique. Tout au long de son séjour, le chien passera successivement de l'état de vivant-personne à celui de vivant-matière. Cette série de morcellements ontologiques de l'animal a pour effet de rendre cohérente toute décision prise à son égard: l'animal que l'on choisira d'euthanasier n'est pas vu comme le même animal que celui que l'on a accueilli dans le refuge quelques semaines plus tôt, et dont on avait toutes les raisons de croire qu'il serait adoptable. Petit à petit, on a pu observer son comportement au sein du refuge, et estimer qu'il ne le sera jamais. Grâce à la séparation des différents états de l'animal, la décision d'euthanasie est moins mal vécue par les équipes. Vient s'ajouter à ce morcellement ontologique, la conscience du flux permanent d'animaux : ce qui pèse dans la décision d'euthanasie est cette certitude qu'il y aura toujours des animaux à faire entrer dans le refuge, et que plutôt d'encombrer les cages avec des animaux qui y passeront sans doute toute leur vie, il vaut mieux libérer de l'espace pour d'autres qui seront adoptés rapidement. La prise en compte de cet état de fait impacte fortement la décision d'euthanasie, et la rend acceptable moralement (sans jamais la banaliser). Le fonctionnement qui vient d'être décrit permet de pointer une donnée essentielle : si l'objectif du refuge est bien de produire des êtres irremplaçables, c'est bien parce que durant leur séjour en son sein, les animaux sont toujours potentiellement remplaçables. Dans son ouvrage La Condition fotale (2004), Luc Boltanski s'intéresse à certains arrangements qui permettent à la pratique de l'avortement d'être moralement acceptée par les femmes. Parmi ceux-ci, la certitude de pouvoir être à nouveau enceinte rend l'avortement tolérable pour celles qui y ont recours (sans jamais être un acte anodin sur le plan psychologique). Le caractère remplaçable du fœetus expulsé est ce qui fait que l'avortement n'est pas totalement vécu comme un infanticide, mais comme une expulsion de matière, de chair. L'être expulsé est humain par la chair, mais son appartenance à l'humanité comme communauté n'a pas été confirmée par la parole, par l'attribution d'un habillage symbolique (un nom, un projet, une place dans la filiation). Il y a donc une distinction entre des êtres remplaçables (humains par la chair) et des êtres singuliers, irremplaçables (confirmés par la parole). Il ne s'agit pas de dire que cette destruction d'un être de chair est très bien vécu par les femmes grâce 
à ce type d'arrangements, mais qu'elle devient moins pesante moralement car on sait que cet être est remplaçable par d'autres êtres semblables - qui pourront devenir irremplaçables par un don de singularité, sur le mode de la parole, lorsque l'engendrement sera mené à son terme. Le lien entre la question de l'avortement et le refuge doit être fait ici. En son sein, les animaux sont débarrassés de tout habillage symbolique : seules comptent leurs singularités corporelles et comportementales. Ils se trouvent donc dans la situation d'être remplacés par d'autres: d'être adoptés et donc reconnus comme des êtres irremplaçables, ou bien euthanasiés. Dès lors, le refuge apparait comme une matrice chargée d'engendrer des animaux singuliers et irremplaçables, mais qui, dans de nombreux cas, ne mène pas à terme cet engendrement. Les mécanismes permettant de réduire les tensions liées à l'élimination d'êtres en cours d'engendrement sont en cela similaires à ceux que l'on observe dans le cas de l'avortement. Ce détour par la question de l'avortement nous permet d'insister sur un point: l'acte d'engendrement est éminemment anthropologique, et il l'est d'autant plus qu'il n'est pas toujours mené à son terme. La valeur de l'accueil d'un nouveau membre de la communauté humaine provient en partie du fait que cet être aurait très bien pu ne pas advenir : l'acte d'engendrement inclue toujours la potentialité de l'avortement, décidé ou subi. Boltanski montre bien que l'avortement est une pratique qui ne se dit pas, ou peu, mais qui pourtant se retrouve dans toutes les cultures, de tout temps. De fait, penser l'engendrement sans l'avortement, ne serait ce que comme potentialité, c'est ne représenter que partiellement le phénomène anthropologique. Il en va de même pour les euthanasies d'animaux : ne pas en parler revient à occulter toute une partie de la dimension anthropologique de ce qu'accomplit le refuge. Penser l'adoption d'animaux de compagnie sans penser l'euthanasie, c'est passer à côté de la dynamique sociale qu'incarnent les refuges.

\section{CONCLUSION}

J'espère avoir montré que, ne serait ce que d'un point de vue scientifique, parler de l'euthanasie des animaux de refuge est essentiel pour comprendre les évolutions récentes des relations humains/animaux. Penser le refuge comme un dispositif de production d'animaux singuliers et personnifiés, compatibles avec les attentes sociales en la matière, est déjà un pas conséquent dans cette compréhension. Mais voir le refuge en tant que matrice qui engendre certains êtres ET en détruit certains autres permet de l'appréhender comme un opérateur de régulation des relations humains/animaux typiquement moderne (au sens de Latour, 1991), veillant à bien séparer ce qui, dans chaque être, relève du social, du symbolique, de la 
culture, et ce qui relève de la matière, du corps, de la nature. Dans ce sens, c'est bel et bien à un objet important de l'anthropologie des mondes occidentaux contemporains que l'on a affaire. Et pour que cette anthropologie soit complète, il faut évoquer l'euthanasie. Récemment, des travaux sociologiques sur l'élevage (Porcher, 2002, 2003; Mouret, 2012) ont cherché à ne pas réduire cette activité à la mise à mort programmée des bêtes, à recentrer le regard sur le don de vie que constitue le fait d'élever des animaux, de s'en occuper au quotidien, et d'en prendre soin. De la même façon, la compréhension du phénomène «animal de compagnie» ne saurait se concentrer que sur les mécanismes de personnification des animaux: les phénomènes de réification, de dégradation (Rémy \& Winance, 2010) sont également à documenter.

Enfin, sur un plan plus politique, il est important de rendre publique la question des euthanasies dans les refuges pour pouvoir engager un débat démocratique autour de la manière dont nous souhaitons gérer l'errance animale en milieu urbain, ou tout simplement la présence d'animaux domestiques sans maîtres dans nos villes. Ce débat n'a jamais vraiment eu lieu à grande échelle. Pour le tenir, les fourrières municipales, les associations de protection animale et les chenils privés devront bien évoquer leurs pratiques, et en faire le bilan. La mort programmée des animaux de refuge devra alors sortir de l'ombre.

Jérôme MICHALON

Docteur en Sociologie et Anthropologie Politique Laboratoire Environnement, Ville, Société (UMR 5600)

Centre Max Weber (UMR 5283)

jerome.michalon@gmail.com

\section{BIBLIOGRAPHIE}

Agulhon M. (1981), «Le sang des bêtes. Le problème de la protection des animaux en France au XIX ${ }^{\mathrm{e}}$ siècle », in Romantisme, n³1, p. 81-109.

Arluke A. (1991), "Coping with euthanasia: A case study of shelter culture», in Journal of the American Veterinary Medicine Association, ${ }^{\circ} 198, \mathrm{p} .1176-1180$. Baldin D. (2014), Histoire des animaux domestiques (XIX ${ }^{e}-X X^{e}$ siècle), Paris, Seuil. Baratay E. (2008), La Société des animaux. De la Révolution à la Libération, Lyon, La Martinière.

Baratay E. (2011), «Chacun jette son chien. De la fin d'une vie au XIX ${ }^{\mathrm{e}}$ siècle », in Romantisme, Vol.2011-3, no 153,p.147-162. 
Boltanski L. (2004), La condition fotale. Une sociologie de l'engendrement et de l'avortement, Paris, Gallimard.

Brisebarre A.-M. Dir. (1998), «Mort et mise à mort des animaux », in Etudes Rurales, no 147-148.

Cohen P. (1984), «La fièvre du samedi après-midi », in Autrement, ${ }^{\circ}$ 56, p. 173-184.

Digard J.-P. (1999), Les français et leurs animaux. Ethnologie d'un phénomène de société, Paris, Hachette Littératures.

Fleury G. (1995), La belle histoire de la S.P.A. de 1845 à nos jours, Paris, Grasset et Flasquelle.

Frank J. \& Carlisle-Frank P. (2003), «Companion Animal Overpopulation : Trends and Results of Major Efforts to Reach a No-Kill Nation », in Annual meeting of the American Sociological Association, Atlanta Hilton Hotel, Atlanta, GA.

Frommer S. S. \& Arluke A. (1999), «Loving Them to Death: Blame-Displacing Strategies of Animal Shelter Workers and Surrenderers », in Society and Animals, Vol. 7, no 1,p. 1-16.

Latour B. (1991), Nous n'avons jamais été modernes. Essai d'anthropologie symétrique, Paris, La Découverte.

Marsh P. (2010), Replacing Myth with Math: Using Evidence-Based Programs to Eradicate Shelter Overpopulation, Selfpublished (http ://www.shelteroverpopulation.org), Concord.

Michalon J. (2013), «Fabriquer l'animal de compagnie. Ethnographie d'un refuge S.P.A.» in Sociologie, Vol. 4, no 2, p. 163-181.

Micoud A. (2010), «Sauvage ou domestique, des catégories obsolètes ?», in Sociétés, Vol. 2, no 108,p. 99-107.

Mouret S. (2012), Élever et tuer des animaux, Paris, Presses Universitaires de France.

Pierre E. (1998), «La souffrance des animaux dans les discours des protecteurs français au XIXe siècle», in Études rurales, no 147-148, p. 81-97.

Porcher J. (2002), Éleveurs et animaux, réinventer le lien, Paris, Presses Universitaires de France.

Porcher J. (2003), La mort n'est pas notre métier, Editions de l'Aube.

Rémy C. \& Winance M. (2010), «Pour une sociologie des «frontières d'humanité », in Politix, Vol. 23, no 90, p. 9-19.

Rowan A. N. \& Williams J. (1992), «Shelters and Pet Overpopulation: A Statistical Black Hole», in Anthrozoos, 1992, Vol. 5, no 3, p. 140-143.

Traïni C. (2011), La cause animale (1820-1980). Essai de sociologie historique, Paris, Presses Universitaires de France.

Jérôme MICHALON - Pourquoi faut-il parler de la mort des animaux de refuge?

Résumé: Cet article part du constat de la méconnaissance actuelle concernant les euthanasies pratiquées par les refuges de protection animale, aussi bien au niveau de leur nombre, de leur justification ou bien de leurs conditions d'exercice. L'auteur cherchera à montrer en quoi cette méconnaissance est dommageable tout autant pour la compréhension de l'évolution récente des 
rapports humains/animaux dans les sociétés occidentales contemporaines, que pour le débat public autour de la gestion des animaux domestiques en ville.

Mots-clés : Protection animale - Euthanasie - Relations humains/animaux - Animaux de compagnie.

Jérôme MICHALON - On the Importance of Talking about Animals Put Down in Shelters.

\begin{abstract}
This article points out the lack of knowledge about the euthanasia practised in animal shelters, in terms both of the number of animals put down, the justification for these acts and their circumstances. The author of this paper shows that this lack of knowledge is harmful for our understanding of recent evolutions in human/animal relationships in contemporary western societies, and of the public debate about pet management within the urban context.
\end{abstract}

Key-words : Animal welfare - Euthanasia - Human/animal relationships Companion animals. 